\title{
Subtleties in the quasi-classical calculation of Hawking radiation
}

\author{
Emil T.Akhmedov* \\ Moscow, B.Cheremushkinskaya, 25, ITEP, Russia 117218 \\ Terry Pilling $\dagger$ \\ Department of Physics, North Dakota State University, Fargo, ND 58105 \\ Douglas Singleton \\ Physics Department, CSU Fresno, Fresno, CA 93740-8031
}

(Dated: November 1, 2018)

\begin{abstract}
The quasi-classical method of deriving Hawking radiation is investigated. In order to recover the original Hawking temperature one must take into account a previously ignored contribution coming from the temporal part of the action. This contribution plus a contribution coming from the spatial part of the action gives the correct temperature.
\end{abstract}

*Electronic address: akhmedov@itep.ru

$\dagger$ Electronic address: Terry.Pilling@ndsu.edu

${ }^{\ddagger}$ Electronic address: dougs@csufresno.edu 
Introduction: By considering a quantum field in a fixed black hole background of mass $M$ one finds [1] that the black hole emits thermal radiation with a temperature $T_{H}=\frac{\hbar}{8 \pi M}$ (we set $k_{B}=c=G=1$ ). The crucial point leading to this result is that the collapse of a star into a black hole changes the vacuum state for quantum fields. The latter process converts zero modes with respect to the vacuum before the collapse into a Planckian spectrum with respect to the vacuum after the collapse. This gives an attractive picture for the origin of black hole radiation. However, this picture does not give a microscopic description of the radiation, which would be helpful e.g. for taking into account the back-reaction of the radiation on the black hole.

The hope is that a quasi-classical "tunneling" process may give such a microscopic description. The "tunneling" picture seeks to describe black hole radiation by pair creation near the black hole horizon with the subsequent tunneling of one of the particles of the pair through the horizon. The tunneling rate is found as the exponent of the imaginary part of the classical action for the particles coming from the vicinity of the horizon.

However, the interpretation of the imaginary contribution to the particle's action as an indication of tunneling is subtle. First, if the pair is created behind the horizon neither of the particles can tunnel through the horizon, because the tunneling process in quantum mechanics is described via the solution of a Cauchy problem and has to be causal, while passing through the horizon is acausal. In quantum mechanics the vacuum remains unchanged which is the reason why we can safely convert a time evolution problem into an eigen-value problem. Second, if the pair is created outside a horizon the time for one of the particles to cross the horizon is infinite for the stationary distant observer. However, this same observer should see the radiation from the black hole in finite time after the collapse. Finally, the description of black hole radiation as pair creation in a strong gravitational field via the production of virtual super-luminal particles does not have an explicit calculation.

In [2] [3] the following idea was proposed to give a physical picture for the quasi-classical tunneling description of black hole radiation: during the pair creation process the horizon shrinks slightly so that the radiated particle already appears outside the horizon. This obviates the problems discussed in the previous paragraph. This method of calculating Hawking radiation has also been shown to give the standard relationship between black hole temperature and entropy [4].

In turn the calculation of the imaginary contribution to the classical action in a black 
hole background via the solution of the Hamilton-Jacobi equation is analogous to the quasi-classical approximation for the calculation of the probability of vacuum decay in an external electro-magnetic field [5]. In this type of calculation applied to a gravitational background one looks for the imaginary contribution to the vacuum decay amplitude, i.e. $\operatorname{Tr} \log \left[\square(g)+m^{2}\right]=\int D x(t) e^{-\frac{i}{\hbar} S(g, x)}$, where $\square(g)$ is the d'Alembertian operator in the background metric $g_{\mu \nu}$ for a particle of mass $m$. On the right hand side we have a path integral over closed paths and $S$ is the action for particles in the gravitational field. In the quasi-classical calculation $(\hbar \rightarrow 0)$ we find the saddle-point approximation for the path integral. The main imaginary contribution comes from the closed paths which cross the horizon going out and back. In other words in the quasi-classical calculation we find an imaginary eigen-value contribution to $\operatorname{Tr} \log \left[\square(g)+m^{2}\right]$.

Hamilton-Jabobi Equations: For a particle, of mass, $m$, the Hamilton-Jacobi equation is

$$
g^{\mu \nu}\left(\partial_{\mu} S\right)\left(\partial_{\nu} S\right)+m^{2}=0
$$

where $g^{\mu \nu}$ is the inverse metric of the background space-time, $S(x)$ is the action of the particle, in terms of which the corresponding scalar field can be written as $\phi(x) \propto$ $\exp \left[-\frac{i}{\hbar} S(x)+\ldots\right]$.

For stationary space-times with a time-like Killing vector, to describe a positive energy state, one can split the action into a time and spatial part

$$
S\left(x^{\mu}\right)=E t+S_{0}(\vec{x})
$$

where $E$ is the particle energy and $x^{\mu}=(t, \vec{x})$. Inserting some particular metric into (1) gives an equation for $S_{0}(\vec{x})$ which has the solution $S_{0}=-\int p_{r} d r$ where $p_{r}$ is the canonical momentum in the background metric. If $S_{0}$ has an imaginary part one can read off the temperature of the radiation as follows: The rate for a quasi-classical process can be written as

$$
\begin{aligned}
\Gamma & \propto \exp \left[-\operatorname{Im} \oint p_{r} d r / \hbar\right]=\exp \left[-\operatorname{Im}\left(\int p_{r}^{\text {Out }} d r-\int p_{r}^{I n} d r\right) / \hbar\right] \\
& =\exp \left[\mp 2 \operatorname{Im} \int p_{r}^{\text {Out,In}} d r / \hbar\right] .
\end{aligned}
$$

The closed path of integration in the first expression goes across the barrier and back (in our case the integration goes around the horizon in the complex $r$ plane). $S_{0}^{\text {Out }, \text { In }}=-\int p_{r}^{\text {Out,In }} d r$ 
are the two different traversal directions across the barrier. The expression in the second line of (3) assumes that $p_{r}^{\text {Out }}=-p_{r}^{\text {In }}$ i.e. crossing the barrier left to right versus right to left only differs by a sign. Strictly one should use $\oint p_{r} d r=\int p_{r}^{\text {Out }} d r-\int p_{r}^{I n} d r$ since only this quantity, not $2 \int p_{r}^{\text {Out,In }} d r$, is invariant under canonical transformations (see e.g. [7] for the discussion in the context of black holes). Thus, only $\oint p_{r} d r$ is a proper observable. It happens that in the black hole background for the metrics which are regular on the horizon $\operatorname{Im} \oint p_{r} d r \neq \pm 2 \operatorname{Im} \int p_{r}^{\text {Out,In }} d r$, because $\int p_{r}^{\text {Out }} d r$ has a non-zero imaginary contribution, while $\int p_{r}^{I n} d r$ does not. Below we consider such a difference in greater detail for the black hole metric in Painlevé coordinates.

In order to obtain a temperature from (3) we associate it with a Boltzmann factor $\Gamma \propto$ $\exp (-E / T)$ so that $T=\frac{E \hbar}{\operatorname{Im} \oint p_{r} d r}$ (note that $T$ is independent of $E$ ).

Spatial Contribution: The $1+1$ Schwarzschild metric is

$$
d s^{2}=-\left(1-\frac{2 M}{r}\right) d t^{2}+\frac{d r^{2}}{\left(1-\frac{2 M}{r}\right)} .
$$

By spherical symmetry the angular part is not important. The above procedure gives

$$
S_{0}= \pm \int_{0}^{+\infty} \frac{d r}{\left(1-\frac{2 M}{r}\right)} \sqrt{E^{2}-m^{2}\left(1-\frac{2 M}{r}\right)}= \pm \int_{0}^{\infty} p_{r}^{\text {In,Out }} d r
$$

It is well known [6] that doing the contour integration for (5) by going around the pole at $r=2 M$ with a semi-circular path gives $\operatorname{Im}\left(S_{0}\right)^{(\text {In }, \text { Out })}= \pm 2 \pi M E$ where $+=I n$ is for the ingoing particle and $-=$ Out is for the outgoing particle. This yields a temperature twice as large as Hawking's original calculation. Initially this disagreement between the quasiclassical approach and other methods was attributed to the "badness" of the Schwarzschild coordinates near the horizon. Below we show that the source of the disagreement comes from a missed contribution coming from the temporal part of the total action, $S\left(x^{\mu}\right)=E t+S_{0}$.

Since $\oint p_{r} d r$ is canonically invariant it will be the same in any coordinate coordinates related to the Schwarzschild coordinates via a canonical transformation and one does not need to re-calculate it in different coordinates. However we mention briefly how $\oint p_{r} d r$ remains fixed in two commonly used coordinate systems: isotropic and Painlevé-Gulstrand. In isotropic coordinates, which are related to Schwarzschild coordinates via $r=\rho(1+M / 2 \rho)^{2}$, one apparently finds [8] $\operatorname{Im} S_{0}^{\text {In,Out }}= \pm 4 \pi M E$ if one treats the isotropic $\rho$ exactly like $r$. However near the horizon the relationship between $r$ and $\rho$ goes like $\rho \simeq \sqrt{r}[6]$. One must apply the transformation to both the integrand and the contour of the integration in $S_{0}$. 
This transforms the semi-circular contour of the Schwarzschild coordinates to a quarter circle contour in the isotropic coordinates. Thus one has $i \frac{\pi}{2} \times$ Residue rather than $i \pi \times$ Residue which again yields $\operatorname{Im} S_{0}^{I n, O u t}= \pm 2 \pi M E$ [6]. In the Painlevé-Gulstrand coordinates, which are related to the Schwarzschild coordinates via $d t^{\prime}=d t+\frac{\sqrt{\frac{2 M}{r}} d r}{1-\frac{2 M}{r}}$ with radial and angular coordinates the same, one finds that only the outgoing path has an imaginary contribution. This happens because a freely falling particle which crosses the horizon does not have any barrier in the Painlevé-Gulstrand coordinates. This leads to $\oint p_{r} d r=\int p_{r}^{\text {Out }} d r-\int p_{r}^{I n} d r=$ $\int p_{r}^{\text {Out }} d r-0=4 \pi M E$. Unlike the Schwarzschild or isotropic coordinates, where both ingoing and outgoing paths contribute equally, here only the outgoing path contributes with twice the magnitude. The net result in all coordinates is $\operatorname{Im} \oint p_{r} d r=4 \pi M E$. Taken by itself this spatial contribution would give a Hawking temperature twice the expected value.

Temporal Contribution: The resolution to this factor of two in the temperature comes from a previously missed, imaginary contribution from the time part of $S\left(x^{\mu}\right)$ i.e. from Et. Thus, we really have a quasi-classical process in two coordinates rather than in one. The reason why there is a non-trivial contribution to the imaginary action coming from the time part is because the " $t$ " coordinate inside the horizon is different from the " $t$ " coordinate outside the horizon. To see the relation between those two " $t$ " coordinates let us use the Kruskal-Szekeres coordinates to go across the horizon.

The Kruskal-Szekeres coordinates $(T, R)$ are related to Schwarzschild $(t, r)$ in the region exterior to the black hole $(r>2 M)$ given by

$$
T=\left(\frac{r}{2 M}-1\right)^{1 / 2} e^{r / 4 M} \sinh \left(\frac{t}{4 M}\right), \quad R=\left(\frac{r}{2 M}-1\right)^{1 / 2} e^{r / 4 M} \cosh \left(\frac{t}{4 M}\right) .
$$

For the interior of the black hole $(r<2 M)$ the relationship is

$$
T=\left(1-\frac{r}{2 M}\right)^{1 / 2} e^{r / 4 M} \cosh \left(\frac{t}{4 M}\right), \quad R=\left(1-\frac{r}{2 M}\right)^{1 / 2} e^{r / 4 M} \sinh \left(\frac{t}{4 M}\right) .
$$

To connect these two patches across the horizon at Schwarzschild $r=2 M$ one needs to "rotate" the Schwarzschild $t$ as $t \rightarrow t-4 M i \frac{\pi}{2}=t-2 \pi i M$ (together with the change $r-2 M \rightarrow 2 M-r)$ and then one sees that $(T, R)$ for the exterior patch given in (6) becomes the $(T, R)$ for the interior patch given in (17). This "rotation" also needs to be taken into account in the time part of the action (i.e. in Et). This leads to an additional imaginary contribution coming from the temporal part of the action. The imaginary part of this temporal contribution is $\operatorname{Im}\left(E \Delta t^{I n, O u t}\right)=-2 \pi M E$. For a round trip this 
will yield $\operatorname{Im}(E \Delta t)=-4 \pi M E$ which is the same magnitude as the spatial contribution $\operatorname{Im} \oint p_{r} d r=4 \pi M E$. Thus the total imaginary contribution in any coordinate frame related to Schwarzschild by a canonical transformation can be written (remembering that $\left.S_{0}=-\int p_{r} d r\right)$

$$
\operatorname{Im}\left(S\left(x^{\mu}\right)\right)=\operatorname{Im}\left(E \Delta t^{O u t}+E \Delta t^{I n}-\oint p_{r} d r\right)=-8 \pi M E,
$$

which yields the canonical Hawking temperature $T_{H}=\frac{\hbar}{8 \pi M}$.

We have shown that the quasi-classical method has a previously overlooked imaginary part coming from the temporal part of the total action. Only by taking this extra contribution into account does one recover the standard Hawking temperature. A similar time contribution must be taken into account when one applies the quasi-classical method to other gravitational backgrounds (e.g. Reissner-Nordstrom, Kerr, Kerr-Newman, deSitter [9]). Note that in the case of Unruh radiation there is no contribution from the time part since the time coordinate does not have a similar change when one crosses from one section of space-time to the other.

Acknowledgments ETA acknowledges discussions with G.Volovik and support from the Agency of Atomic Energy of Russian Federation and a CSU Fresno Provost Grant.

[1] S.W. Hawking, Comm. Math. Phys. 43, 199 (1975).

[2] P. Kraus and F. Wilczek, Nucl. Phys. B 437231 (1995). E. Keski-Vakkuri and P. Kraus, Nucl. Phys. B 491249 (1997).

[3] M.K. Parikh and F. Wilczek, Phys. Rev. Lett. 85, 5042 (2000); M.K. Parikh Int. J. Mod. Phys. D 132351 (2004).

[4] T. Pilling, Phys. Lett. B660, 402 (2008).

[5] J. Schwinger, Phys. Rev. 82, 664 (1951).

[6] E.T. Akhmedov, V. Akhmedova and D. Singleton, Phys. Lett. B, 642124 (2006); E.T. Akhmedov, V. Akhmedova, D. Singleton, and T. Pilling, Int. J. Mod. Phys. A, 221705 (2007).

[7] B. D. Chowdhury, Pramana 70, 593 (2008)

[8] M. Angheben, M. Nadalini, L. Vanzo, and S. Zerbini, JHEP, 0505:014 (2005)

[9] V. Akhmedova, T. Pilling, A. de Gill, and D. Singleton, Phys. Lett. B, 666269 (2008) 